\title{
PROREGULAR SEQUENCES, LOCAL COHOMOLOGY, AND COMPLETION
}

\author{
PETER SCHENZEL
}

\begin{abstract}
As a certain generalization of regular sequences there is an investigation of weakly proregular sequences. Let $M$ denote an arbitrary $R$-module. As the main result it is shown that a system of elements $\underline{x}$ with bounded torsion is a weakly proregular sequence if and only if the cohomology of the $\breve{C}$ ech complex $\check{C}_{\underline{x}} \otimes M$ is naturally isomorphic to the local cohomology modules $H_{\mathfrak{a}}^{i}(M)$ and if and only if the homology of the co-Čech complex $\operatorname{RHom}\left(\breve{C}_{\underline{x}}, M\right)$ is naturally isomorphic to $\mathrm{L}_{i} \Lambda^{\mathfrak{a}}(M)$, the left derived functors of the $\mathfrak{a}$-adic completion, where a denotes the ideal generated by the elements $\underline{x}$. This extends results known in the case of $R$ a Noetherian ring, where any system of elements forms a weakly proregular sequence of bounded torsion. Moreover, these statements correct results previously known in the literature for proregular sequences.
\end{abstract}

\section{Introduction}

Let $R$ denote a commutative ring $R$ and a an ideal of $R$, Then the local cohomology functor $\Gamma_{\mathfrak{a}}$ is defined as the subfunctor of the identity functor such that

$$
\Gamma_{\mathfrak{a}}(M)=\{m \in M: \operatorname{Supp} R m \subseteq V(\mathfrak{a})\}
$$

for an $R$-module $M$. Let $H_{\mathfrak{a}}^{i}$ denote the $i$-th local cohomology functor, i.e. the $i$-th right derived functor of $\Gamma_{\mathfrak{a}}$. In the case $\mathfrak{a}$ is generated by a system of elements $\underline{x}=x_{1}, \ldots, x_{r}$ the local cohomology is closely related to the Čech complex $\check{C}_{\underline{x}}:=\otimes_{i=1}^{r} \check{C}_{x_{i}}$, where $\check{C}_{x_{i}}$ is defined as the mapping cone of the natural homomorphism $R \rightarrow R_{x_{i}}$ of $R$-modules.

At the early times of local cohomology, see [9, Exposé II] or [10, Theorem D], it was shown that there are functorial isomorphims

$$
H_{\mathfrak{a}}^{i}(M) \simeq H^{i}\left(\check{C}_{\underline{x}} \otimes M\right), \quad i \in \mathbf{Z},
$$

for any $R$-module $M$ provided $R$ is a Noetherian ring. This was generalized in [1, Proposition 3.1.1] and [8] to the case of $\underline{x}=x_{1}, \ldots, x_{r}$ a proregular sequence in an arbitrary commutative ring $R$. It turned out that this is not correct. To this end J. Lipman suggested the notion of a weakly proregular sequence.

Received November 11, 1999; in revised form November 13, 2000. 
A system of elements $\underline{x}$ is called a weakly proregular sequence whenever for each integer $n>0$ there is an $m \geq n$ such that the natural homomorphism of the Koszul homology

$$
H_{i}\left(\underline{x}^{m}\right) \rightarrow H_{i}\left(\underline{x}^{n}\right)
$$

is zero for each $i \geq 1$, see 2.3 . It follows that in a Noetherian ring $R$ any sequence of elements forms a weakly proregular sequence. Moreover, a regular sequence is also a weakly proregular sequence.

Originally the notion of a proregular sequence was introduced by Greenlees and May, see [8, Definition 1.8], in order to study the left derived functors $\mathrm{L}_{i} \Lambda^{\mathrm{a}}$ of the $\mathfrak{a}$-adic completion $\Lambda^{\mathfrak{a}}=\lim \left(R / \mathfrak{a}^{n} \otimes \cdot\right)$.

There is a large amount of research articles about local cohomology. Not so much is known about the functors $\mathrm{L}_{i} \Lambda^{\mathfrak{a}}$. The main sources for their study are [1], [8], [13], and [17]. By the work of Greenlees and May [8] it turns out that the completion is closely related to a certain dual of the Čech complex, namely $\operatorname{RHom}\left(\check{C}_{\underline{x}}, M\right)$ for an $R$-module $M$. This was extended also to the study of formal schemes and non-Noetherian schemes in [3].

Because $\breve{C}_{\underline{x}}$ is a bounded complex of flat $R$-modules it is not necessary to work in the derived category in order to elaborate on $\check{C}_{\underline{x}} \otimes M$. This is no longer the case for RHom $\left(\check{C}_{\underline{x}}, M\right)$. In the derived category we may represent it by

$$
\operatorname{Hom}\left(L_{\underline{x}}, M\right), \quad \operatorname{Hom}\left(\check{C}_{\underline{x}}, I\right), \quad \text { resp. } \operatorname{Hom}\left(L_{\underline{x}}, I\right),
$$

where $L_{\underline{x}} \stackrel{\sim}{\longrightarrow} \check{C}_{\underline{x}}$ resp. $M \stackrel{\sim}{\longrightarrow} I$ denotes a free resolution of $\check{C}_{\underline{x}}$ resp. an injective resolution of $M$. See Section 4 for an explicit construction of $L_{\underline{x}}$, a bounded complex of free $R$-modules. Let $X$ denote a complex of $R$-modules. One of the main results of the paper is the following result:

Theorem 1.1. Let $R$ be commutative ring. Let $\underline{x}=x_{1}, \ldots, x_{r}$ denote a system of elements of $R$ and $\mathfrak{a}=\underline{x} R$. Suppose that $R$ is of bounded $x_{i} R$-torsion for $i=1, \ldots, r$. Then the following conditions are equivalent:

(i) $\underline{x}$ is a weakly proregular sequence.

(ii) $H^{i}\left(\check{C}_{\underline{x}} \otimes I\right)=0$ for each $i \neq 0$ and each injective $R$-module $I$.

(iii) $H_{i}\left(\operatorname{RHom}\left(\check{C}_{\underline{x}}, F\right)\right)=0$ for each $i \neq 0$ and each flat $R$-module $F$.

(iv) There is a functorial isomorphism

$$
\mathrm{R} \Gamma_{\mathfrak{a}}(X) \approx \check{C}_{\underline{x}} \otimes X
$$

in the derived category.

(v) There is a functorial isomorphism

$$
\operatorname{RHom}\left(\check{C}_{\underline{x}}, X\right) \approx \mathrm{L}^{\mathfrak{a}}(X)
$$


in the derived category, provided $X$ is a bounded complex.

This corrects several results shown in [1], [8], [9], and [10]. It was extended to the case of schemes, see [1] and [2]. In particular, it is noteworthy to say that there is no finiteness condition on the cohomology of the complex $X$ in (iv) and (v). The proof requires several steps in Section 3 and 4. As a main technical tool we need the Koszul complexes and cocomplexes. A corollary of these investigations is the following result about weakly proregular sequences, analogous to a corresponding result for regular sequences:

Proposition 1.2. Let $R$ denote a commutative ring. Let $\underline{x}=x_{1}, \ldots, x_{r}$ be a system of elements of $R$. Then the following conditions are equivalent:

(i) $\underline{x}$ is a weakly proregular sequence.

(ii) $\underline{y}$ is a weakly proregular sequence for a system of elements $\underline{y}=y_{1}, \ldots, y_{s}$ such that $\operatorname{Rad} \underline{x} R=\operatorname{Rad} \underline{y} R$.

(iii) For each $i \neq 0$ the Koszul homology modules $\left\{H_{i}\left(\underline{x}^{n}\right)\right\}_{n \in \mathrm{N}}$ are pro-zero.

Here pro-zero means that for each $n$ there is an $m \geq n$ such that the natural homomorphism $H_{i}\left(\underline{x}^{m}\right) \rightarrow H_{i}\left(\underline{x}^{n}\right)$ is zero. Note that for a Noetherian ring the claim in (iii) was shown by Grothendieck, see [9, Exposé II, Lemme 9] and [10, Lemma 2.5].

For a complex of $R$-modules $X$ we freely use the existence of $F \stackrel{\sim}{\longrightarrow} X$, a flat resp. $X \stackrel{\sim}{\rightarrow} I$, an injective resolution of $X$. The existence of such a resolution was proved by Spaltenstein, see [18]. Another approach was developed by Avramov and Foxby, see [4]. See also Weibel's paper [20, Appendix] for a short account to this subject. Moreover, we refer also to Foxby's forthcoming book [6] for all the technical details about derived functors and categories developed by Hartshorne in [12].

Another representative of $\operatorname{RHom}\left(\check{C}_{\underline{x}}, X\right), X$ an arbitrary complex, in the derived category is

$$
\operatorname{Hom}\left(\check{C}_{\underline{x}}, I\right) \simeq \lim _{\leftarrow} K_{\bullet}\left(\underline{x}^{n}, I\right),
$$

where $X \stackrel{\sim}{\rightarrow} I$ denotes an injective resolution. The Koszul complexes $K \bullet\left(\underline{x}^{n} ; I\right)$ satisfy an important homological property.

Proposition 1.3. Let $\underline{x}=x_{1}, \ldots, x_{r}$ denote a sequence of elements of $R$ such that $R$ is of bounded $x_{i} R$-torsion for $i=1, \ldots, r$. Let $I$ be a complex of injective $R$-modules. Then the tower of inverse systems of complexes $\left\{K_{\bullet}\left(\underline{x}^{n} ; I\right)\right\}_{n \in \mathrm{N}}$ satisfies the Mittag-Leffler condition.

The paper is organized as follows. In Section 2 we start with the study of weakly proregular sequences. Section 3 is devoted to the investigation about local cohomology. Section 4 contains the results about completions. 
The author is grateful to Hans-Bjørn Foxby for some stimulating questions concerning the results of 1.1. He also thanks J. Lipman for the discussion concerning a gap in a preliminary version of the paper and suggesting the notion of a weakly proregular sequence.

\section{Weakly Proregular Sequences}

For the next couple of results we need the Koszul homology and cohomology. For a system of elements $\underline{x}=x_{1}, \ldots, x_{r}$ let $K_{\bullet}(\underline{x})$ resp. $K^{\bullet}(\underline{x})$ denote the Koszul complex resp. the Koszul cocomplex. For an arbitrary complex of $R$ modules $X$ we define

$$
K_{\bullet}(\underline{x} ; X)=K_{\bullet}(\underline{x}) \otimes X \quad \text { and } \quad K^{\bullet}(\underline{x} ; X)=\operatorname{Hom}\left(K_{\bullet}(\underline{x}), X\right),
$$

see $[5, \S 9]$. There are the following Koszul duality isomorphisms

$$
K^{\bullet}(\underline{x} ; X) \simeq K^{\bullet}(\underline{x}) \otimes X \quad \text { and } \quad K_{\bullet}(\underline{x} ; X) \simeq \operatorname{Hom}\left(K^{\bullet}(\underline{x}), X\right) .
$$

Denote by $H_{i}(\underline{x} ; X)$ resp. $H^{i}(\underline{x} ; X)$ the homology resp. cohomology of the corresponding complexes.

For an integer $n$ put $\underline{x}^{n}=x_{1}^{n}, \ldots, x_{r}^{n}$. By the construction of the complexes there are natural homomorphisms

$$
K_{\bullet}\left(\underline{x}^{m} ; X\right) \rightarrow K_{\bullet}\left(\underline{x}^{n} ; X\right) \quad \text { and } \quad K^{\bullet}\left(\underline{x}^{n} ; X\right) \rightarrow K^{\bullet}\left(\underline{x}^{m} ; X\right)
$$

for all $m \geq n>0$ such that $\left\{K_{\bullet}\left(\underline{x}^{n} ; X\right)\right\}$ resp. $\left\{K^{\bullet}\left(\underline{x}^{n} ; X\right)\right\}$ forms an inverse resp. a direct system of complexes. Clearly they induce inverse systems resp. direct systems on the homology resp. cohomology modules.

In the following put $\underline{x}_{j}, 1 \leq j \leq r$, for the subsystem of elements $x_{1}, \ldots, x_{j}$. In particular $\underline{x}_{0}=\emptyset$ and $\underline{x}_{r}=\underline{x}$.

Definition 2.1. The inverse system of $R$-modules $\left\{M_{n}, \phi_{n}^{m}\right\}$ is called pro-zero if for each $n \in \mathrm{N}$ there is an $m \geq n$ such that the map

$$
\phi_{n}^{m}: M_{m} \rightarrow M_{n}
$$

is the zero homomorphism.

This definition is useful in order to elaborate on inverse limits as follows by the next observation.

Proposition 2.2. a) Let $\left\{M_{n}, \phi_{n}^{m}\right\}$ denote an inverse system that is pro-zero. Then

$$
\lim _{\longleftarrow} M_{n}=\lim _{\leftarrow}^{1} M_{n}=0 .
$$


b) Let $0 \rightarrow\left\{M_{n}^{\prime}\right\} \rightarrow\left\{M_{n}\right\} \rightarrow\left\{M_{n}^{\prime \prime}\right\} \rightarrow 0$ denote a short exact sequence of inverse systems of $R$-modules. Then the middle inverse system is pro-zero if and only if the two outside ones are pro-zero.

Proof. For the proof of a) note that $\lim _{\leftarrow} M_{n}$ and $\lim ^{1} M_{n}$ are kernel and cokernel of the following homomorphism

$$
\Phi: \prod_{n \in \mathrm{N}} M_{n} \rightarrow \prod_{n \in \mathrm{N}} M_{n}, \quad\left(x_{n}\right) \mapsto\left(x_{n}-\phi_{n}^{n+1}\left(x_{n+1}\right)\right),
$$

see e.g. [19, Corollary 3.5.4]. In the case $\left\{M_{n}, \phi_{n}^{m}\right\}$ is pro-zero it is easily seen that $\Phi$ is an isomorphism, i.e. $\operatorname{Ker} \Phi=\operatorname{Coker} \Phi=0$.

The statement in b) is obviously true, see [10, Remark 2, p. 24].

The previous statements prepare the following definition; in a certain sense it is a generalization of the notion of a regular sequence.

Definition 2.3. A system of elements $\underline{x}=x_{1}, \ldots, x_{r}$ of $R$ is called a weakly proregular sequence if for each $i=1, \ldots, r$ the inverse system of Koszul homology modules $\left\{H_{i}\left(\underline{x}^{n}\right)\right\}$ is pro-zero, i. e. for each $n \in \mathbf{N}$ there is an $m \geq n$ such that the natural homomorphism $H_{i}\left(\underline{x}^{m}\right) \rightarrow H_{i}\left(\underline{x}^{n}\right)$ is the zero homomorphism.

The next lemma provides the first couple of properties related to the homological applications we will study in the following.

Lemma 2.4. Let $\underline{x}=x_{1}, \ldots, x_{r}$ denote a system of elements of $R$. Then the following conditions are equivalent:

(i) $\underline{x}$ is a weakly proregular sequence.

(ii) $\left\{H_{i}\left(\underline{x}^{n} ; F\right)\right\}$ is pro-zero for all $i \neq 0$ and each flat $R$-module $F$.

(iii) $\lim _{\rightarrow} H^{i}\left(\underline{x}^{n} ; I\right)=0$ for all $i \neq 0$ and for each injective $R$-module $I$.

Proof. While the implication (ii) $\Rightarrow$ (i) is trivial we first show the reverse implication in order to see that the first two conditions are equivalent. This follows because

$$
H_{i}\left(\underline{x}^{n}\right) \otimes F \simeq H_{i}(\underline{x} ; F)
$$

for all $i$ since $F$ is a flat $R$-module.

Now let us prove (i) $\Rightarrow$ (iii). Since $I$ is an injective $R$-module

$$
H^{i}\left(\operatorname{Hom}\left(K_{\bullet}\left(\underline{x}^{n}\right), I\right)\right) \simeq \operatorname{Hom}\left(H_{i}\left(\underline{x}^{n}\right), I\right)
$$

for all $i$. Therefore

$$
\lim _{\rightarrow} H^{i}\left(\underline{x}^{n} ; I\right) \simeq \lim _{\rightarrow} \operatorname{Hom}\left(H_{i}\left(\underline{x}^{n}\right), I\right) .
$$


By the assumption $\left\{H_{i}\left(\underline{x}^{n}\right)\right\}$ is pro-zero for $i \neq 0$. Whence the direct limit $\lim _{\rightarrow} H^{i}\left(\underline{x}^{n} ; I\right)$ vanishes, as required.

In order to complete the proof we have to show that (iii) $\Rightarrow$ (i). Let $f$ : $H_{i}\left(\underline{x}^{n}\right) \rightarrow I$ denote an injection into an injective $R$-module $I$. Then

$$
f \in \operatorname{Hom}\left(H_{i}\left(\underline{x}^{n}\right), I\right) \simeq H^{i}\left(\underline{x}^{n} ; I\right)
$$

since $I$ is an injective $R$-module. Because of the assumption we have the vanishing $\underset{\lim }{\longrightarrow} H^{i}\left(\underline{x}^{n} ; I\right)=0$. So there must be an integer $m \geq n$ such that the

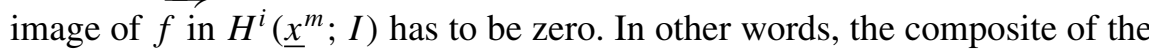
map

$$
H_{i}\left(\underline{x}^{m}\right) \rightarrow H_{i}\left(\underline{x}^{n}\right) \stackrel{f}{\rightarrow} I
$$

is zero. Since $f$ is an injection it follows that the first map has to be zero.

As an application of Lemma 2.4 let us derive a few more properties of weakly proregular sequences, similar to those of a regular sequence.

Corollary 2.5. Let $\underline{x}=x_{1}, \ldots, x_{r}$ denote a system of elements of $R$. Then the following conditions are equivalent:

(i) $\underline{x}$ is a weakly proregular sequence.

(ii) There is an $m>0$ such that $\underline{x}^{m}$ is a weakly proregular sequence.

(iii) For any permutation $\sigma$ of $\{1, \ldots, r\}$ the sequence $x_{\sigma(1)}, \ldots, x_{\sigma(r)}$ is a weakly proregular sequence.

Proof. The equivalence of the first and the third condition follows since the corresponding Koszul complexes are isomorphic. In order to complete the proof one has to show that (ii) $\Rightarrow$ (i). To this end note that

$$
\lim _{\rightarrow} H^{i}\left(\underline{x}^{n} ; I\right) \simeq \lim _{\longrightarrow} H^{i}\left(\underline{x}^{m n} ; I\right)=0
$$

for any injective $R$-module. Then the claim follows by 2.4 .

The following notion of a proregular sequence was introduced by Greenlees and May, see [8, Definition 1.8] It was also studied in [1, Section 3] and [7]. We shall relate it to the definition of the weakly proregular sequence of 2.3.

Definition 2.6. A system of elements $\underline{x}=x_{1}, \ldots, x_{r}$ of $R$ is called a proregular sequence if for each $i=1, \ldots, r$ and each $n>0$ there is an $m \geq n$ such that

$$
\left(x_{1}^{m}, \ldots, x_{i-1}^{m}\right) R:_{R} x_{i}^{m} \subseteq\left(x_{1}^{n}, \ldots, x_{i-1}^{n}\right) R:_{R} x_{i}^{m-n} .
$$


In the case $R$ is a Noetherian ring for a fixed integer $n$ the increasing sequence of ideals

$$
\left(x_{1}^{n}, \ldots, x_{i-1}^{n}\right):_{R} x_{i}^{m-n}, m \geq n,
$$

will stabilize. Therefore in a Noetherian ring $R$ any sequence of elements forms a proregular sequence.

It follows by the definition that $\underline{x}$ is a proregular sequence if and only if for each $i=1, \ldots, r$ and each $n>0$ there exists an $m \geq n$ such that the multiplication map

$$
\begin{aligned}
\left(x_{1}^{m}, \ldots, x_{i-1}^{m}\right) R:_{R} x_{i}^{m} /\left(x_{1}^{m}, \ldots, x_{i-1}^{m}\right) R & \\
& \stackrel{x_{i}^{m-n}}{\longrightarrow}\left(x_{1}^{n}, \ldots, x_{i-1}^{n}\right) R:_{R} x_{i}^{n} /\left(x_{1}^{n}, \ldots, x_{i-1}^{n}\right) R
\end{aligned}
$$

is zero. This indicates the homological flavour of this notion related to that of a weakly proregular sequence.

Lemma 2.7. Let $\underline{x}=x_{1}, \ldots, x_{r}$ denote a system of elements of $R$. Suppose that it is a proregular sequence. Then it is also a weakly proregular sequence.

Proof. We proceed by induction on $r$. For $r=0$ there is nothing to prove. Put $y=x_{r+1}$. Then the Koszul homology provides the following diagram

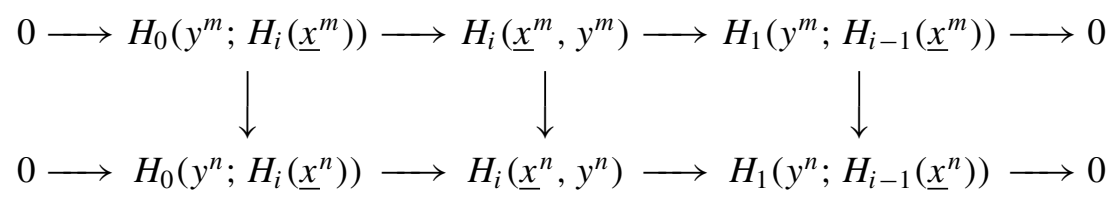

for each $i \in \mathbf{Z}$ and any pair of integers $m \geq n$. The modules at the first vertical map are derived by the following commutative diagram

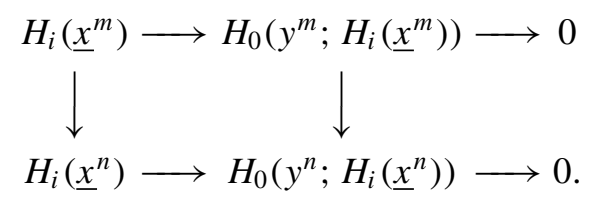

By virtue of $2.2 \mathrm{~b}$ ) and the inductive hypothesis it follows that the first vertical map of the first diagram above is pro-zero for each $i \neq 0$.

The modules on the last vertical map of the diagram above are derived by the following commutative diagram

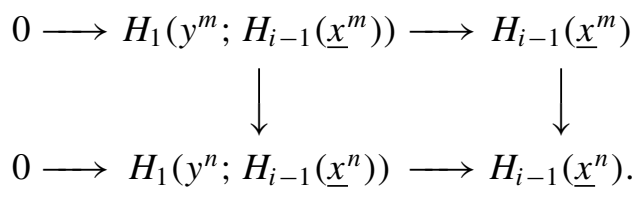


By the same argument as above the vertical map at the first place is pro-zero for all $i \neq 1$. In the case $i=1$ we have

$$
H_{1}\left(y^{n} ; H_{0}\left(\underline{x}^{n}\right)\right) \simeq \underline{x}^{n} R:_{R} y^{n} / \underline{x}^{n} R .
$$

Therefore, by the assumption the vertical homomorphism is also pro-zero in this case. Then by $2.2 \mathrm{~b}$ ) the first diagram above implies that $\left\{H_{i}\left(\underline{x}^{n}, y^{n}\right)\right\}$ is essentially zero for each $i \neq 0$, completing the inductive step.

It is noteworthy to say that a weakly proregular sequence is - in general - not proregular. The following example was kindly communicated by J. Lipman to the author, see [2]. Let $R=\prod_{n>0} \mathbf{Z} / 2^{n} \mathbf{Z}$ and $x=(2,2,2, \ldots)$. Then it follows that $H_{i}\left(\underline{x}^{n}\right)=0$ for the sequence $\underline{x}=x, 1$ and all $i \in \mathrm{Z}$. Therefore $\underline{x}$ is weakly proregular. But it is not proregular, while $1, x$ is so. Whence the example shows also that a proregular sequence is not permutable without any additional assumption.

\section{Local cohomology and Čech complexes}

Let $\underline{x}=x_{1}, \ldots, x_{r}$ denote a sequence of elements of a commutative ring $R$. Then the direct limit of the Koszul cocomplexes $\underset{\lim }{\longrightarrow} K^{\bullet}\left(\underline{x}^{n}\right)$ is called the Čech complex $\check{C}_{\underline{x}}$ of $R$ with respect to $\underline{x}$. It is easily seen that $\breve{C}_{\underline{x}} \simeq \otimes_{i=1}^{r} \check{C}_{x_{i}}$, where $\check{C}_{x_{i}}$ is the complex

$$
\check{C}_{x_{i}}: \ldots \rightarrow 0 \rightarrow R \rightarrow R_{x_{i}} \rightarrow 0 \rightarrow \ldots,
$$

see e.g. [15, Section 1.1] for the details. In particular $\check{C}_{\underline{x}}$ is a bounded complex of flat $R$-modules.

On the other hand let $\mathfrak{a}$ be an ideal of $R$. Then $\Gamma_{\mathfrak{a}}$ denotes the section functor with respect to $a$. That is, $\Gamma_{\mathfrak{a}}$ is the subfunctor of the identity functor given by

$$
\Gamma_{\mathfrak{a}}(M)=\{m \in M: \operatorname{Supp} R m \subseteq V(\mathfrak{a})\}
$$

for an $R$-module $M$. It extends to a functor on complexes of $R$-modules. Let $X \stackrel{\sim}{\rightarrow} I$ be an injective resolution of $X$, see [4] resp. [18], for the details. Then define $\mathrm{R} \Gamma_{\mathfrak{a}}(X)=\Gamma_{\mathfrak{a}}(I)$, the right derived functor of $\Gamma_{\mathfrak{a}}$ in the derived category. In fact the construction is independent on the particular choice of $I$, see [12] for the details.

Proposition 3.1. Let $\underline{x}=x_{1}, \ldots, x_{r}$ be a system of elements of $R$ and $\mathfrak{a}=\underline{x} R$ the ideal generated by it. For a complex $X$ of $R$-modules there is a functorial morphism

$$
\mathrm{R} \Gamma_{\mathfrak{a}}(X) \rightarrow \check{C}_{\underline{x}} \otimes X
$$

in the derived category. 
Proof. Let $X \stackrel{\sim}{\rightarrow} I$ denote an injective resolution of $X$, see [4] resp. [18]. Then $\mathrm{R} \Gamma_{\mathfrak{a}}(X)$ resp. $\check{C}_{\underline{x}} \otimes X$ are - in the derived category - represented by $\Gamma_{\mathfrak{a}}(I)$ resp. by $\check{C}_{\underline{x}} \otimes I$. In order to prove the claim we have to show that there is a natural injection $\Gamma_{\mathfrak{a}}(I) \rightarrow \check{C}_{\underline{x}} \otimes I$. Since $\Gamma_{\mathfrak{a}}\left(I^{n}\right)=\operatorname{Ker}\left(I^{n} \rightarrow I^{n} \otimes \check{C}_{x}^{1}\right)$ for each $n \in \mathbf{Z}$ the following diagram

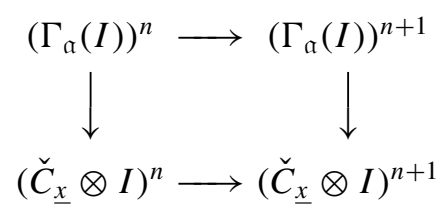

commutes. Here the vertical homomorphisms map $\Gamma_{\mathfrak{\alpha}}\left(I^{n}\right)$ to $\check{C}_{x}^{0} \otimes I^{n}=I^{n}$ by the natural inclusion. So there is an injection

$$
\Gamma_{\mathfrak{a}}(I) \rightarrow \check{C}_{\underline{x}} \otimes I
$$

of complexes. This proves the morphism of the claim. It is easily seen functorial and independent on the particular choice of $I$.

Now it is natural to ask whether the morphism of Proposition 3.1 is an isomorphism. In particular this yields an isomorphism

$$
H_{\mathfrak{a}}^{i}(X) \simeq H^{i}\left(\check{C}_{\underline{x}} \otimes X\right)
$$

for all $i$. This was shown to be true whenever $R$ is a Noetherian ring, see [9, Exposé II] and [10].

THEOREM 3.2. Let $\underline{x}=x_{1}, \ldots, x_{r}$ be a system of elements of $R$ and $\mathfrak{a}=\underline{x} R$. Then the following conditions are equivalent:

(i) $\underline{x}$ is a weakly proregular sequence.

(ii) $H^{i}\left(\check{C}_{\underline{x}} \otimes I\right)=0$ for each $i \neq 0$ and each injective $R$-module $I$.

(iii) For each complex $X$ the functorial morphism

$$
\mathrm{R} \Gamma_{\mathfrak{a}}(X) \rightarrow \check{C}_{\underline{x}} \otimes X
$$

is an isomorphism in the derived category.

Proof. The equivalence of (i) and (ii) is an easy consequence of Lemma 2.4. Note that $\underset{\longrightarrow}{\longrightarrow}$ is exact and $\underline{\lim } K^{\bullet}\left(\underline{x}^{n}\right) \simeq \check{C}_{\underline{x}}$. The implication (iii) $\Rightarrow$ (ii) holds

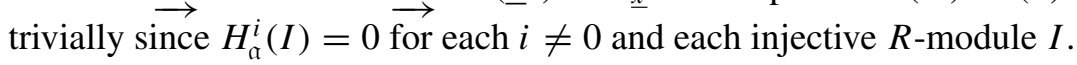

Now let us prove (ii) $\Rightarrow$ (iii). To this end take an injective resolution $X \stackrel{\sim}{\longrightarrow} I$ of $X$, see [4] resp. [18]. The $j$-th column $\breve{C}_{\underline{x}} \otimes I^{j}$ of the double complex

$$
\check{C}_{\underline{x}}^{i} \otimes I^{j}, \quad 0 \leq i \leq m, \quad j \in \mathrm{Z},
$$


is - by the assumption - an injective resolution of $\Gamma_{\mathfrak{a}}\left(I^{j}\right)$, so that the inclusion

$$
\Gamma_{\mathfrak{a}}(I) \rightarrow \check{C}_{\underline{x}} \otimes I
$$

induces an isomorphism in cohomology. This completes the proof.

The previous result was originated by [1] and [8]. In fact, Theorem 3.2 shows the equivalence of the conditions (i), (ii), and (iv) of Theorem 1.1.

There is an application concerning another property of proregular sequences.

Corollary 3.3. Let $\underline{x}=x_{1}, \ldots, x_{r}$ resp. $y=y_{1}, \ldots, y_{s}$ denote two systems of $R$ such that $\operatorname{Rad} \underline{x} R=\operatorname{Rad} y R$. Then $\underline{x}$ is a weakly proregular sequence if and only if $\underline{y}$ is a weakly proregular sequence.

Proof. Let $\mathfrak{a}=\underline{x} R$ and $\mathfrak{b}=\underline{y} R$. Then $\mathrm{R} \Gamma_{\mathfrak{a}}(X)=\mathrm{R} \Gamma_{\mathfrak{b}}(X)$ for any complex of $R$-modules $X$ since $\operatorname{Rad} \overline{\mathfrak{a}}=\operatorname{Rad} \mathfrak{b}$. Therefore the claim follows by the Theorem 3.2 and 2.4 .

As mentioned above, in a Noetherian ring $R$ any system of elements forms a weakly proregular sequence since it is proregular. Conversely it would be of some interest to characterize those commutative rings for which any finite system of elements forms a weakly proregular sequence.

The proof of Proposition 1.2 is now a consequence of Corollary 3.3 together with Lemma 2.4.

In the following we will continue with a result concerning the composite of two section functors. It is well known in the case of a Noetherian ring $R$.

Corollary 3.4. Let $\underline{x}, y=x_{1}, \ldots, x_{r}, y_{1}, \ldots, y_{s}$ denote a weakly proregular sequence consisting of the two weakly proregular subsystems $\underline{x}, \underline{y}$. Put $\mathfrak{a}=\underline{x} R$ resp. $\mathfrak{b}=\underline{y} R$. Then there is a functorial isomorphism

$$
\left.\mathrm{R} \Gamma_{\mathfrak{a}}\left(\mathrm{R} \Gamma_{\mathfrak{b}}(X)\right) \approx \mathrm{R} \Gamma_{\mathfrak{a}+\mathfrak{b}}(X)\right)
$$

for a complex of $R$-modules $X$.

Proof. Since $\underline{x}, \underline{y}$ forms a weakly proregular sequence it follows that

$$
\left.\mathrm{R} \Gamma_{\mathfrak{a}+\mathfrak{b}}(X)\right) \approx \check{C}_{\underline{x}, \underline{y}} \otimes X,
$$

see 3.2. Moreover both $\underline{x}$ and $\underline{y}$ form a weakly proregular sequence by the assumption. Furthermore, by the construction of the Čech complex we have the isomorphism $\check{C}_{\underline{x}, \underline{\underline{y}}} \simeq \check{C}_{\underline{x}} \otimes \check{C}_{\underline{y}}$. So the claim is a consequence of 3.2 and the associativity of the tensor product. 
In the particular case that $s=1$ and $y$ consists of a single element $y$ there is a short exact sequence useful for an inductive increase of the number of elements in local cohomology.

Corollary 3.5. Let $\underline{x}=x_{1}, \ldots, x_{r}, y$, and $\underline{x}, y$ denote weakly proregular sequences. For each $i \in \mathrm{Z}$ there is a functorial short exact sequence

$$
0 \rightarrow H_{y R}^{1}\left(H_{\mathfrak{a}}^{i-1}(X)\right) \rightarrow H_{\mathfrak{a}+y R}^{i}(X) \rightarrow H_{y R}^{0}\left(H_{\mathfrak{a}}^{i}(X)\right) \rightarrow 0,
$$

where $X$ denotes an arbitrary complex of $R$-modules.

Proof. By the fact that $\underline{x}, y$, and $\underline{x}, y$ form a weakly proregular sequence resp. we may compute the right derived functor of the corresponding section functors by the $\breve{C}$ ech complexes. Now $\check{C}_{\underline{x}, y}$ is by construction the mapping cone of the natural homomorphism $\check{C}_{\underline{x}} \rightarrow \check{C}_{\underline{x}} \otimes R_{y}$. So the short exact sequence of complexes

$$
0 \rightarrow \check{C}_{\underline{x}} \otimes R_{y}[-1] \rightarrow \check{C}_{\underline{x}, y} \rightarrow \check{C}_{\underline{x}} \rightarrow 0
$$

provides the exact sequences of the statement. Note that the localization $R_{y}$ is exact.

In the case of a Noetherian ring $R 3.5$ has been shown in [15, Corollary 1.4]. The property of $y$ being a weakly proregular sequence is equivalent to saying that $y R$ is of bounded $y R$-torsion, see the definition in 4.2 .

\section{Completion and co-Čech complexes}

In a certain sense - which will become more precise in the following - completion is a construction dual to the local cohomology. While the local cohomology modules are studied in several research papers not so much is known about the derived functors of the completion.

The most significant papers to the present research are - first of all - the work of Greenlees and May, see [8], and the papers [1], [13], and [17]. For an ideal a of $R$ let $\Lambda^{\mathfrak{a}}$ denote the a-adic completion functor $\lim \left(R / \mathfrak{a}^{n} \otimes \cdot\right)$. For an arbitrary complex $X$ of $R$-modules let $F \stackrel{\sim}{\longrightarrow} X$ denote $\overleftarrow{\leftarrow}$ flat resolution of $X$, see [4] resp. [18] for its existence.

DEFINITION 4.1. In the derived category the left derived functor $\operatorname{L} \Lambda^{\mathfrak{a}}(X)$ of $X$ is defined by $\Lambda^{\mathfrak{a}}(F)$, where $F \stackrel{\sim}{\longrightarrow} X$ denotes a flat resolution.

In fact, this construction is functorial and independent of the choice of the particular resolution $F$, see [1], [8], and [17] for the details.

Let $\underline{x}=x_{1}, \ldots, x_{r}$ denote a system of elements of the ring $R$. Let $X$ be an arbitrary complex of $R$-modules. Then the complex, the so-called co-Čech complex,

$$
\operatorname{RHom}\left(\check{C}_{\underline{x}}, X\right)
$$


in a certain sense the dual of $\check{C}_{\underline{x}} \otimes X$, is of a great importance related to the completion functor. While the complex $\check{C}_{\underline{x}} \otimes X$ is well-defined in the category of modules, the co-Čech complex is an object in the derived category. It is represented by $\operatorname{Hom}\left(\check{C}_{\underline{x}}, I\right)$, where $X \stackrel{\sim}{\longrightarrow} I$ denotes an injective resolution of $X$. Another representative of $\operatorname{RHom}\left(\check{C}_{\underline{x}}, X\right)$ will be constructed in the following.

Let $x \in R$ denote an element. The naturally defined short exact sequence

$$
0 \rightarrow R[T] \stackrel{x T-1}{\longrightarrow} R[T] \rightarrow R_{x} \rightarrow 0
$$

provides a free resolution of $R_{x}$ as an $R$-module. Let $P_{x}$ denote the truncated resolution consisting of $R[T]$ in degree 0 and -1 and zero elsewhere. Let $L_{x}$ denote the mapping cone of the natural homomorphism of complexes $R \rightarrow P_{x}$. Then it follows by the construction that $L_{x} \stackrel{\sim}{\longrightarrow} \check{C}_{x}$ is a free resolution of the Čech complex $\check{C}_{x}$.

Now let $\underline{x}=x_{1}, \ldots, x_{r}$ denote a system of elements of $R$. Then define

$$
L_{\underline{x}}=\otimes_{i=1}^{r} L_{x_{i}} .
$$

Clearly $L_{\underline{x}} \stackrel{\sim}{\longrightarrow} \check{C}_{\underline{x}}$ is a free resolution of the Čech complex $\check{C}_{\underline{x}}$. Therefore, in the derived category the complex $\operatorname{RHom}\left(\check{C}_{\underline{x}}, X\right)$ is represented by each of the following complexes

$$
\operatorname{Hom}\left(\check{C}_{\underline{x}}, I\right) \stackrel{\sim}{\longrightarrow} \operatorname{Hom}\left(L_{\underline{x}}, I\right) \text { and } \operatorname{Hom}\left(L_{\underline{x}}, X\right) \stackrel{\sim}{\longrightarrow} \operatorname{Hom}\left(L_{\underline{x}}, I\right),
$$

where $X \stackrel{\sim}{\rightarrow} I$ denotes an injective resolution of $X$.

We continue here with another property of a proregular sequence. It requires the following definition concerning the torsion properties.

Definition 4.2. Let a denote an ideal of $R$. Then $R$ is said to be of bounded a-torsion if the increasing sequence $\left\{0:_{R} \mathfrak{a}^{m}\right\}_{m \in \mathrm{N}}$ stabilizes.

Note that whenever $\underline{x}=x_{1}, \ldots, x_{r}$ denotes a proregular sequence, $R$ is of bounded $x_{1} R$-torsion. In the case of $R$ a Noetherian ring it is of bounded a-torsion for any ideal $a$ of $R$.

Now note that $\operatorname{RHom}\left(\check{C}_{\underline{x}}, X\right)$ is - in the derived category - also represented by

$$
\operatorname{Hom}\left(\check{C}_{\underline{x}}, I\right) \simeq \operatorname{Hom}\left(\lim _{\longrightarrow} K^{\bullet}\left(\underline{x}^{n}\right), I\right) \simeq \lim _{\leftarrow} K \bullet\left(\underline{n}^{n} ; I\right),
$$

where $X \stackrel{\sim}{\longrightarrow} I$ denotes an injective resolution of $X$. Here we are interested in the complex $\lim _{\leftarrow} K_{\bullet}\left(\underline{x}^{n} ; I\right)$ and its cohomology.

THEOREM 4.3. Let $\underline{x}=x_{1}, \ldots, x_{r}$ denote a system of elements of $R$. Then the following conditions are equivalent:

(i) $R$ is of bounded $x_{j}$-torsion for each $j=1, \ldots, r$. 
(ii) For each injective $R$-module I and each $j=1, \ldots, r$ the multiplication map $I \stackrel{x_{j}}{\longrightarrow} I$ becomes stable, i.e. there is an integer $n$ such that $x_{j}^{n} I=$ $x_{j}^{m}$ I for all $m>n$.

(iii) The tower of inverse systems of complexes $\left\{K_{\bullet}\left(\underline{x}^{n} ; I\right)\right\}$ satisfies the Mittag-Leffler condition.

Proof. First we show the implication (i) $\Rightarrow$ (ii). To this end let $x \in R$ denote an arbitrary element. For each pair of integers $m \geq n$ there is the following diagram induced by multiplications

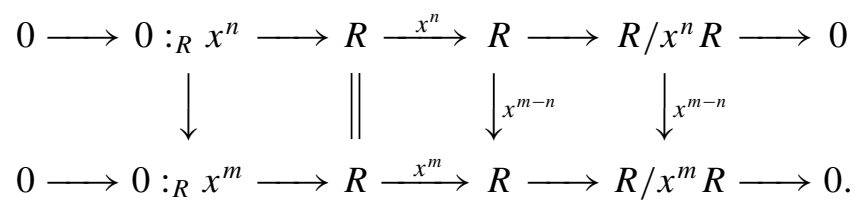

Since $I$ is an injective $R$-module it induces - as easily seen - a commutative diagram of the following type

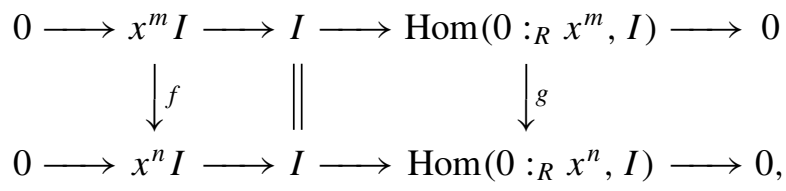

where $f$ is injective and $g$ is surjective. Hence, the snake lemma provides that $\operatorname{Ker} g=$ Coker $f$. In case $R$ is of bounded $x R$-torsion condition (ii) is satisfied. Note that Ker $g=0$ in this situation.

We proceed by an induction on $r$ in order to prove (ii) $\Rightarrow$ (iii). For $r=0$ there is nothing to show. Suppose the claim is true for $r$. Now put $y=x_{r+1}$. We shall prove the claim for the system of $r+1$ elements $\underline{x}, y$.

For each $n$ and $m \geq n$ the natural commutative diagram of Koszul complexes

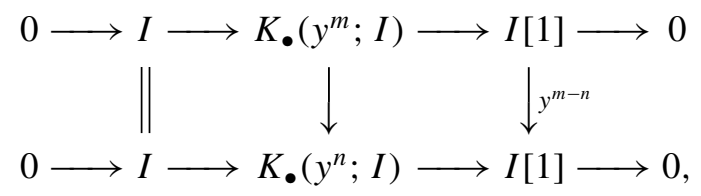

induces the following commutative diagram

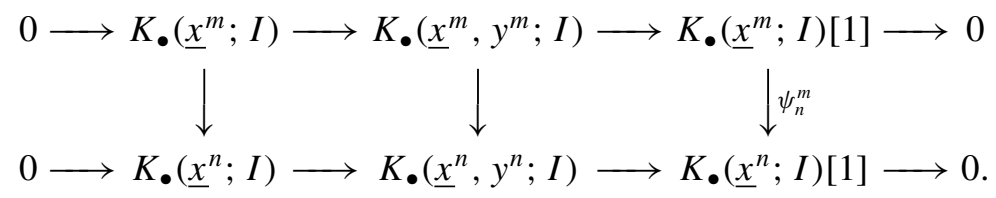

The vertical map $\psi_{n}^{m}$ at the right is the composite of the natural map

$$
\phi_{n}^{m}: K_{\bullet}\left(\underline{x}^{m} ; I\right) \rightarrow K_{\bullet}\left(\underline{x}^{n} ; I\right)
$$


with the multiplication by $y^{m-n}$ on $K \cdot\left(\underline{x}^{n} ; I\right)$. The tower of inverse systems of complexes on the left satisfies the Mittag-Leffler condition by the induction hypothesis.

We claim now that the tower of inverse systems of complexes at the right satisfies the Mittag-Leffler condition too. To this end put $K^{n}=K_{i}\left(\underline{x}^{n} ; I\right)$. Then we have to show that for each $i$ and each $n \geq 1$ there is an integer $m$ such that the image of the homomorphisms $\psi_{n}^{m+s}: K^{m+s} \rightarrow K^{n}$ are the same for all $s \geq 1$. By the inductive hypothesis this is true for the homomorphisms $\phi_{n}^{m}: K^{m} \rightarrow K^{n}$, i.e. for a given $n$ there is an $m \geq n$ such that $\operatorname{Im} \phi_{n}^{m+s}=\operatorname{Im} \phi_{n}^{m}$ for each $s \geq 0$.

For the fixed integer $m$ consider now the multiplication map $\rho_{y^{s}}: K^{m} \rightarrow$ $K^{m}$ by $y^{s}$. Since $K^{m}$ is an injective $R$-module there exists - by the assumption - an integer $t$ such that $\operatorname{Im} \rho_{y^{t+s}}=\operatorname{Im} \rho_{y^{t}}$ for each $s \geq 1$. Therefore

$$
\begin{aligned}
\operatorname{Im} \psi_{n}^{m+s+t} & =y^{m-n+s+t} \phi_{n}^{m+s+t}\left(K^{n+s+t}\right)=y^{m-n+s+t} \phi_{n}^{m}\left(K^{m}\right) \\
& =\phi_{n}^{m}\left(y^{m-n+s+t} K^{m}\right)=\phi_{n}^{m}\left(y^{m-n+t} K^{m}\right) \\
& =y^{m-n+t} \phi_{n}^{m}\left(K^{m}\right)=y^{m-n+t} \phi_{n}^{m+t}\left(K^{m+t}\right) \\
& =\operatorname{Im} \psi_{n}^{m+t}
\end{aligned}
$$

for all $s \geq 0$.

Now the above commutative diagram is split exact in each homological degree. Both of the towers of complexes on the left and on the right satisfy the Mittag-Leffler condition. By [11, 13.2.1] it follows that the tower of complexes in the middle satisfies the Mittag-Leffler condition too. This finishes the proof of (iii).

Finally we have to show the implication (ii) $\Rightarrow$ (i). To this end let $K$ denote an injective co-generator of the category of $R$-modules, see [14, p. 79]. That is, for each $R$-module $M$ and an element $0 \neq m \in M$ there is a homomorphism $f \in$ $\operatorname{Hom}(M, K)$ such that $f(m) \neq 0$. By the assumption (iii) the inverse system $\left\{K_{1}\left(\underline{x}^{n} ; K\right)\right\}$ satisfies the Mittag-Leffler condition. Because of $K_{1}\left(\underline{x}^{n} ; K\right) \simeq$ $\oplus_{i=1}^{r} K$ and because of the homomorphism $K_{1}\left(\underline{x}^{m} ; K\right) \rightarrow K_{1}\left(\underline{x}^{n} ; K\right)$ which is the multiplication by $x_{j}^{m-n}$ on the $j$-th component, $j=0, \ldots, r$, it turns out that the multiplication map by $x_{j}^{m-n}$ on $K$ is stable. By the above commutative diagram it follows that

$$
\operatorname{Hom}\left(0:_{R} x_{j}^{m}, K\right)=\operatorname{Hom}\left(0:_{R} x_{j}^{n}, K\right)
$$

for a large $n$ and all $m>n$. The corresponding short exact sequence implies that $\operatorname{Hom}\left(0:_{R} x_{j}^{m} / 0:_{R} x_{j}^{n}, K\right)=0$. Since $K$ is an injective co-generator it follows that $0:_{R} x_{j}^{m}=0:_{R} x_{j}^{n}$, i.e. $R$ is of bounded $x_{j}$-torsion. 
The previous result has an important application concerning the computation of the homology of the complex $\lim _{\leftarrow} K \cdot\left(\underline{x}^{n} ; I\right)$ for a complex of injective $R$-modules $I$.

Corollary 4.4. Let I denote a complex of injective $R$-modules. Let $\underline{x}=$ $x_{1}, \ldots, x_{r}$ denote a system of elements such that $R$ is of bounded $x_{j}$-torsion for each $j=1, \ldots, r$. Then there is a short exact sequence

$$
0 \rightarrow \lim ^{1} H_{i+1}\left(\underline{x}^{n} ; I\right) \rightarrow H_{i}\left(\lim _{\leftarrow} K_{\bullet}\left(\underline{x}^{n} ; I\right)\right) \rightarrow \lim _{\leftarrow} H_{i}\left(\underline{x}^{n} ; I\right) \rightarrow 0
$$

for each $i \in \mathbf{Z}$.

Proof. In order to show the claim take the homomorphism of complexes

$$
\Phi: \prod_{n \in \mathrm{N}} K_{\bullet}\left(\underline{x}^{n} ; I\right) \rightarrow \prod_{n \in \mathrm{N}} K_{\bullet}\left(\underline{x}^{n} ; I\right)
$$

as considered in the proof of 2.2. Because of the Mittag-Leffler condition shown in 4.3 it induces a short exact sequence of complexes

$$
0 \rightarrow \lim _{\leftarrow} K_{\bullet}\left(\underline{x}^{n} ; I\right) \rightarrow \prod_{n \in \mathbb{N}} K_{\bullet}\left(\underline{x}^{n} ; I\right) \rightarrow \prod_{n \in \mathbb{N}} K_{\bullet}\left(\underline{x}^{n} ; I\right) \rightarrow 0 .
$$

The long exact cohomology sequence induces the short exact sequences of the statement, see also [8] for some more details.

The proof of Proposition 1.3 follows now by the result shown in 4.4. The short exact sequence on the cohomology is of some importance in the following.

There is a functorial homomorphism $K_{\bullet}\left(\underline{x}^{n} ; X\right) \rightarrow X \otimes R / \mathfrak{a}^{n}$ for each $n \geq 0$. Whence, for each $i$ it induces a functorial homomorphism

$$
H_{i}\left(\lim _{\leftarrow} K_{\bullet}\left(\underline{x}^{n} ; I\right)\right) \rightarrow \mathrm{L} \Lambda_{i}^{\mathfrak{a}}(X),
$$

where $X \stackrel{\sim}{\longrightarrow} I$ denotes an injectice resolution of $X$. Recall that $\lim _{\leftarrow} K_{\bullet}\left(\underline{x}^{n} ; I\right)$ is another representative of $\operatorname{RHom}\left(\check{C}_{\underline{x}}, X\right)$, where $X \stackrel{\sim}{\longrightarrow} I$ is an injective resolution of $X$.

TheOREM 4.5. Let $\underline{x}=x_{1}, \ldots, x_{r}$ denote a system of elements of $R$. Suppose that $R$ is of bounded $x_{j}$-torsion for $j=1, \ldots, r$. Then the following conditions are equivalent:

(i) $\underline{x}$ is a weakly proregular sequence.

(ii) For each $R$-module $M$ with $M \stackrel{\sim}{\longrightarrow} I$ its injective resolution the homomorphism

$$
H_{i}\left(\lim _{\leftarrow} K_{\bullet}\left(\underline{x}^{n} ; I\right)\right) \simeq \mathrm{L}_{i} \Lambda^{\mathfrak{a}}(M)
$$

is a functorial isomorphism. 
(iii) For each bounded complex $X$ the functorial morphism

$$
\operatorname{RHom}\left(\check{C}_{\underline{x}}, X\right) \approx \mathrm{L} \Lambda^{\mathfrak{a}}(X)
$$

is an isomorphism in the derived category.

(iv) $H_{i}\left(\operatorname{RHom}\left(\check{C}_{\underline{x}}, F\right)\right)=0$ for each $i \neq 0$ and each flat $R$-module $F$.

Proof. Firstly we show the implication (i) $\Rightarrow$ (ii). Since for each $i \in Z$ there is a functorial isomorphism

$$
H_{i}\left(\lim _{\leftarrow} K_{\bullet}\left(\underline{x}^{n} ; I\right)\right) \simeq H_{i}\left(\operatorname{Hom}\left(P_{\underline{x}}, M\right)\right)=: H_{i}(M)
$$

it will be enough to show the following steps:

1. $H_{0}(M) \simeq \mathrm{L}_{0} \Lambda^{\mathfrak{a}}(M)$.

2. $H_{i}(F)=0$ for each $i \neq 0$ and each flat $R$-module $F$.

3. $\left\{H_{i}\right\}_{i \geq 0}$ forms a connected sequence of functors.

The statement in 3. is true because $P_{x}$ is a bounded complex of free $R$-modules such that $\operatorname{Hom}\left(P_{\underline{x}}, \cdot\right)$ is a covariant functor that preserves quasi-isomorphisms. In order to prove 2 . note that for each $i$ there is a short exact sequence

$$
0 \rightarrow \lim ^{1} H_{i+1}\left(\underline{x}^{n} ; J\right) \rightarrow H_{i}(F) \rightarrow \lim _{\leftarrow} H_{i}\left(\underline{x}^{n} ; J\right) \rightarrow 0,
$$

where $F \stackrel{\sim}{\longrightarrow} J$ denotes an injective resolution, see 4.3. Since $H_{i}\left(\underline{x}^{n} ; F\right) \simeq$ $H_{i}\left(\underline{x}^{n} ; J\right)$ and $\underline{x}$ forms a weakly proregular sequence the inverse system $\left\{H_{i}\left(\underline{x}^{n} ; F\right)\right\}$ is pro-zero, see 2.4. Therefore

$$
H_{i}(F)= \begin{cases}0 & \text { if } i \neq 0, \\ \Lambda^{\mathfrak{a}}(F) & \text { if } i=0,\end{cases}
$$

which proves 2. Note that $H_{0}\left(\underline{x}^{n} ; F\right) \simeq F / \underline{x}^{n} F$ for each $n>0$.

So the claim in 1. remains to prove. As shown above it is true for a flat $R$ module $F$. Let $F_{1} \rightarrow F_{0} \rightarrow M \rightarrow 0$ be a resolution of $M$ by free $R$-modules $F_{i}, i=0,1$. Then it induces a commutative diagram

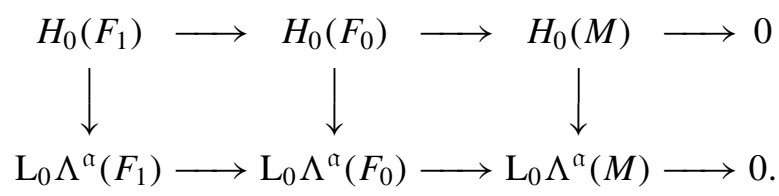

Note that $H_{-1}(\cdot)=0$, as easily seen. Therefore $H_{0}(M) \simeq \mathrm{L}_{0} \Lambda^{\mathfrak{a}}(M)$, as required. 
Now the implication (ii) $\Rightarrow$ (iii) is a consequence of the way-out techniques by Hartshorne, see [12, Chapter I, § 7]. More precisely for $n \in Z$ an integer let

$$
\begin{aligned}
& \sigma_{>n}: \ldots \rightarrow 0 \rightarrow \operatorname{Im} d_{X}^{n} \rightarrow X^{n+1} \rightarrow X^{n+2} \rightarrow \ldots \quad \text { and } \\
& \sigma_{\geq n}: \ldots \rightarrow 0 \rightarrow \operatorname{Coker} d_{X}^{n-1} \rightarrow X^{n+1} \rightarrow X^{n+2} \rightarrow \ldots
\end{aligned}
$$

Then there is a quasi-isomorphism $\sigma_{>n-1} \stackrel{\sim}{\longrightarrow} \sigma_{\geq n}$ and a short exact sequence

$$
0 \rightarrow H^{n}(X)[-n] \rightarrow \sigma_{\geq n} \rightarrow \sigma_{>n-1} \rightarrow 0 .
$$

Then we show by descending induction on $n$ that

$$
\operatorname{RHom}\left(\check{C}_{\underline{x}}, \sigma_{>n}\right) \approx \operatorname{L} \Lambda^{\mathfrak{a}}\left(\sigma_{>n}\right)
$$

in the derived category. For $n$ sufficiently large $\sigma_{>n}$ is the zero complex. So the claim is certainly true. Because of the assumption in (ii) the above short exact sequence provides the claim for $\sigma_{\geq n}$. Since $\sigma_{>n-1} \stackrel{\sim}{\longrightarrow} \sigma_{\geq n}$ is a quasiisomorphism and both functors preserve quasi-isomorphisms the claim is true for $\sigma_{>n-1}$.

Next note that (iii) $\Rightarrow$ (iv) follows since $\mathrm{L}_{i} \Lambda^{\mathfrak{a}}(F)=0$ for each $i \neq 0$ and a flat $R$-module $F$. Finally we have to show the implication (iv) $\Rightarrow$ (i) in order to finish the proof.

To this end let $I$ be an arbitrary injective $R$-module. Let $K$ denote an injective co-generator of the category of $R$-modules, see [14, p. 79]. Because $\operatorname{Hom}(I, K)$ is a flat $R$-module the assumption in (ii) implies that

$$
H_{i}\left(\operatorname{RHom}\left(\check{C}_{\underline{x}}, \operatorname{Hom}(I, K)\right)\right)=0 \quad \text { for each } i \neq 0 .
$$

Because $\operatorname{RHom}\left(\check{C}_{\underline{x}}, \operatorname{Hom}(I, K)\right)$ is represented by $\operatorname{Hom}\left(\check{C}_{\underline{x}} \otimes I, K\right)$ and because $K$ is an injective $R$-module it follows that

$$
0=\operatorname{Hom}\left(H^{i}\left(\check{C}_{\underline{x}} \otimes I\right), K\right) \quad \text { for all } \quad i \neq 0 .
$$

Therefore $H^{i}\left(\check{C}_{\underline{x}} \otimes I\right)=0$ for each $i \neq 0$ and each injective $R$-module $I$. By Theorem 3.2 this completes the proof.

It is an open problem to the author whether (iii) in Theorem 4.5 holds for any complex, similar to the result for the local cohomology in Theorem 3.2, see [1] for various results in this direction. It is true in the case of a Noetherian ring $R$, as shown by different methods in [16].

Finally we mention that Theorem 4.5 proves the equivalence of the statements (i), (iii), and (v) of Theorem 1.1 of the introduction. 
CoROllary 4.6. Let $\underline{x}, \underline{y}=x_{1}, \ldots, x_{r}, y_{1}, \ldots, y_{s}$ denote a weakly proregular sequence consisting of the two weakly proregular subsystems $\underline{x}, y$. Suppose that $R$ is of bounded $x_{i} R$-torsion for $i=1, \ldots, r$ and is of bounded $y_{j}$-torsion for $j=1, \ldots, s$. Put $\mathfrak{a}=\underline{x} R$ resp. $\mathfrak{b}=y R$. Then there is $a$ functorial isomorphism

$$
\left.\mathrm{L} \Lambda^{\mathfrak{a}+\mathfrak{b}}(X)\right) \approx \mathrm{L} \Lambda^{\mathfrak{a}}\left(\mathrm{L} \Lambda^{\mathfrak{b}}(X)\right)
$$

for a bounded complex of $R$-modules $X$.

Proof. Let $X \stackrel{\sim}{\longrightarrow} I$ denote an injective resolution of $X$. Then $\left.\operatorname{L} \Lambda^{\mathfrak{a}+\mathfrak{b}}(X)\right)$ is represented by $\operatorname{Hom}\left(\check{C}_{x, y}, I\right)$ in the derived category, see 4.5 . But now we have that $\check{C}_{\underline{x}, \underline{y}} \simeq \check{C}_{\underline{x}} \otimes \check{C}_{\underline{y}}$. The adjunction formula provides the isomorphism

$$
\operatorname{Hom}\left(\check{C}_{\underline{x}, \underline{y}}, I\right) \simeq \operatorname{Hom}\left(\check{C}_{\underline{x}}, \operatorname{Hom}\left(\check{C}_{\underline{y}}, I\right)\right) .
$$

Furthermore both of the sequences $\underline{x}$ and $y$ form a weakly proregular sequence and $\operatorname{Hom}\left(\check{C}_{y}, I\right)$ is a complex of injective $R$-modules. Whence by 4.5 the second complex in the above isomorphism represents $\operatorname{L} \Lambda^{\mathfrak{a}}\left(\mathrm{L} \Lambda^{\mathfrak{b}}(X)\right)$ in the derived category. This completes the arguments.

In the case of $s=1$, i.e. $y$ consists of a single element $y$ there is a short exact sequence for computing the left derived functors of the completion inductively. The proof of the following corollary is a little more complicated than the corresponding result for the local cohomology shown in 3.5.

COROLlaRY 4.7. Let $\underline{x}$ and $\underline{x}, y$ denote weakly proregular sequences. Suppose that $R$ is of bounded $y R$-torsion and of bounded $x_{i} R$-torsion for $i=1, \ldots, r$. For each $i \in \mathbf{Z}$ there is a functorial short exact sequence

$$
0 \rightarrow \mathrm{L}_{0} \Lambda^{y R}\left(\mathrm{~L}_{i} \Lambda^{\mathfrak{a}}(X)\right) \rightarrow \mathrm{L}_{i} \Lambda^{\mathfrak{a}+y R}(X) \rightarrow \mathrm{L}_{1} \Lambda^{y R}\left(\mathrm{~L}_{i-1} \Lambda^{\mathfrak{a}}(X)\right) \rightarrow 0,
$$

where $X$ denotes a bounded complex of $R$-modules.

Proof. Let $M$ denote an $R$-module. Then we first observe that $\mathrm{L}_{i} \Lambda^{y R}(M)$ $=0$ for all $i \neq 0,1$. Because $R$ is of bounded $y$-torsion and because $\mathrm{L} \Lambda^{y R}(M)$ is represented by $\operatorname{Hom}\left(\check{C}_{y}, I\right)$, where $M \stackrel{\sim}{\longrightarrow} I$ denotes an injective resolution of $M$. Then this claim follows by view of the homology sequence of

$$
0 \rightarrow I \rightarrow \operatorname{Hom}\left(\check{C}_{y}, I\right) \rightarrow \operatorname{Hom}\left(R_{y}, I\right)[1] \rightarrow 0 .
$$

To this end recall that $H_{i}(I)=H_{i}\left(\operatorname{Hom}\left(R_{y}, I\right)\right)=0$ for all $i>0$.

Now consider the free resolution $L_{\underline{x}}$ of the Čech complex $\check{C}_{\underline{x}}$ as defined at the beginning of this section. Then the derived functors of the completion 
may be represented by $\operatorname{Hom}\left(L_{\underline{x}}, X\right)$. By the adjunction formula we have the isomorphism of complexes

$$
\operatorname{Hom}\left(L_{\underline{x}, y}, X\right) \simeq \operatorname{Hom}\left(L_{y}, \operatorname{Hom}\left(L_{\underline{x}}, X\right)\right),
$$

note that $L_{\underline{x}, y} \simeq L_{\underline{x}} \otimes L_{y}$. This yields the following spectral sequence for the homology modules

$$
E_{i j}^{2}=\mathrm{L}_{i} \Lambda^{y R}\left(\mathrm{~L}_{j} \Lambda^{\mathfrak{a}}(X)\right) \Rightarrow E_{i+j}^{\infty}=\mathrm{L}_{i+j} \Lambda^{\mathfrak{a}+y R}(X) .
$$

Because of $E_{i j}^{2}=0$ for all $i \neq 0,1$ it degenerates partially to the short exact sequences of the statement.

An inductive argument provides that $\mathrm{L}_{i} \Lambda^{\mathfrak{a}}(X)=0$ for all $i>r$, the number of elements of $\underline{x}$. A more detailed study of the largest integer $i$ such that $\mathrm{L}_{i} \Lambda^{\mathfrak{a}}(X) \neq 0$ will appear in a forthcoming article by the author.

\section{REFERENCES}

1. Alonso Tarrîo, L., Jeremias López, A., Lipman, J., Local homology and cohomology of schemes, Ann. Sci. École Norm. Sup. (4) 30 (1997), 1-39.

2. Alonso Tarrîo, L., Jeremias López, A., Lipman, J., Local homology and cohomology of schemes, Corrections.

3. Alonso Tarrîo, L., Jeremias López, A., Lipman, J., Studies in duality on Noetherian formal schemes and non-Noetherian ordinary schemes, Cont. Math. 244, Amer. Math. Soc., Providence, 1999.

4. Avramov, L. L., Foxby, H.-B., Homological dimensions of unbounded complexes, J. Pure Appl. Algebra 71 (1991), 129-155.

5. Bourbaki, N., Algèbre, Chap. X: Algébre homologique, Masson, Paris, 1980.

6. Foxby, H.-B., Hyperhomological Algebra and Commutative Rings, preliminary version of the first part, Univ. of Copenhagen, 1996.

7. Greenlees, J. P. C., Augmentation ideals of equivariant cohomology rings, Topology 37 (1998), $1313-1323$.

8. Greenlees, J. P. C., May, J. P., Derived functors of the I-adic completion and local homology, J. Algebra 149 (1992), 438-453.

9. Grothendieck, A., Cohomologie locale des faisceaux cohérents et théorèms de Lefschetz locaux et globaux, SGA 2, North-Holland, Amsterdam, 1962.

10. Grothendieck, A., Local cohomology, notes by R. Hartshorne, Lecture Notes in Math. 20 (1966).

11. Grothendieck, A., Dieudonné, J., Élements de Géomètrie Algébrique III, Inst. Hautes Études Sci. Publ. Math. 11, 1961.

12. Hartshorne, R., Residues and duality, Lecture Notes in Math. 41 (1967).

13. Matlis, E., The Koszul complex and duality, Comm. Algebra 1 (1974), 87-144.

14. Rotman, J. J., An Introduction to Homological Algebra, Academic Press, 1979.

15. Schenzel, P., On the use of local cohomology in algebra and geometry. In: Six Lectures in Commutative Algebra, Proceed. Summer School on Commutative Algebra at Centre de Recerca Matemàtica, (Ed.: J. Elias, J. M. Giral, R. M. Miró-Roig, S. Zarzuela), Progr. Math. 166 (1998), 241-292. 
16. Schenzel, P., Duality Theorems, in preparation.

17. Simon, A.-M., Some homological properties of complete modules, Math. Proc. Cambr. Philos. Soc. 108 (1990), 231-246.

18. Spaltenstein, N., Resolutions of unbounded complexes, Compositio Math. 65 (1988), 121154.

19. Weibel, C., An Introduction to Homological Algebra, Cambr. Univ. Press, 1994.

20. Weibel, C., Cyclic homology for schemes, Proc. Amer. Math. Soc. 124 (1996), 1655-1662.

MARTIN-LUTHER-UNIVERSITÄT HALLE-WITTENBERG

FACHBEREICH MATHEMATIK UND INFORMATIK

D-06 099 HALLE (SAALE)

GERMANY

E-mail: schenzel@mathematik.uni-halle.de 